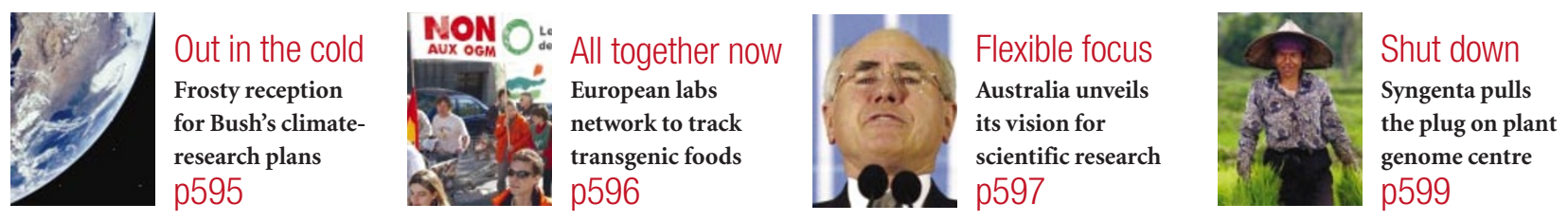

\title{
NASA roadmap charts route to a quarter-century of exploration
}

Tony Reichhardt, Washington

NASA is making preliminary plans for a multibillion-dollar programme to study black holes, dark matter and other cosmic exotica from spaceborne observatories.

Called 'Beyond Einstein', the initiative would be modelled on the agency's Origins programme, which focuses on galaxy and planet formation and the search for life in the Universe.

Like Origins and 'Living With a Star', which covers solar-terrestrial physics, Beyond Einstein is being designed as a coherent framework that would provide a rationale for the support of its scientific field over many years. It would include large space-based observatories, smaller 'Einstein Probes', costing up to $\$ 500$ million each, plus funding for further scientific research and technological development. The plan is set out in a soonto-be-published 25-year strategic roadmap written by scientific advisers to NASA's existing Structure and Evolution of the Universe (SEU) programme.

NASA could request fresh funding for the initiative as early as the 2005 fiscal year, according to Anne Kinney, director of the agency's astronomy and physics division. She told an SEU advisory committee in

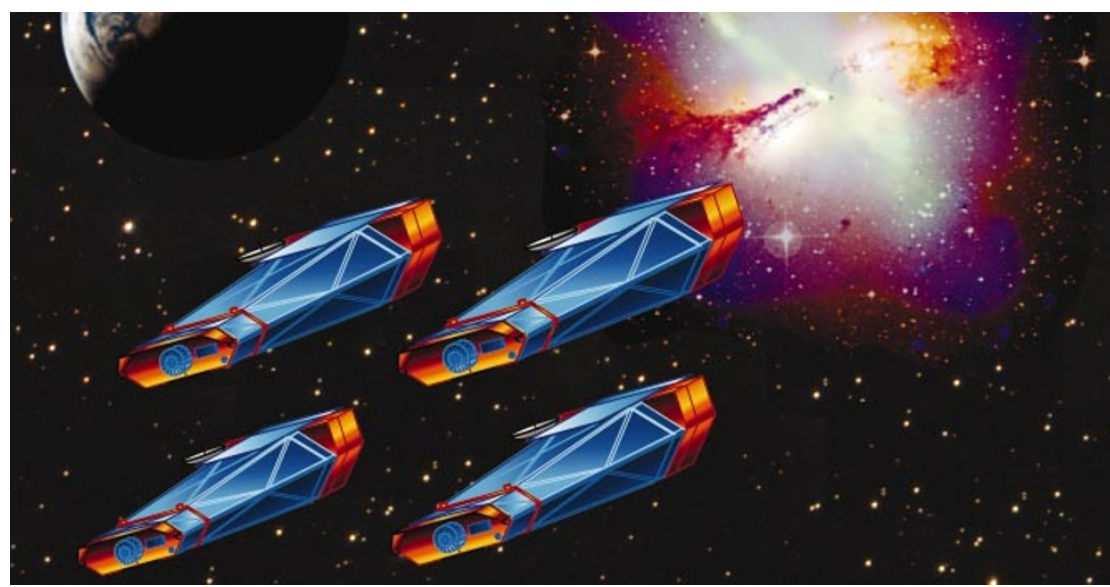

Search across space: Constellation-X will spearhead NASA's programme to study black holes.

Washington on 3 December that Beyond Einstein is NASA chief scientist Ed Weiler's highest-priority new initiative. If approved by the White House and Congress, the programme would probably be funded at a similar level to Origins - several hundred million dollars a year.

Beyond Einstein would build on the work of current spacecraft to address three main research questions: what powered the initial inflation of the Universe following the Big Bang, what happens at the edge of a black hole, and what is dark energy?

The authors of the SEU roadmap, led by Sterl Phinney, a theoretical astrophysicist at the California Institute of Technology, considered two recent National Academy of Sciences reports in shaping their plan: a decadal review of priorities for astronomy missions, and another report on the interface between

\section{Canada stops Harvard's oncomouse in its tracks}

\section{Erika Check, Washington}

The Canadian Supreme Court has turned down a far-reaching patent application on a genetically modified mouse - setting Canada's intellectual property law on a strikingly independent path.

Harvard University had applied to patent the methods for making a transgenic mouse that is highly susceptible to cancer - the socalled 'oncomouse' - in Canada, the United States, Europe and Japan. The university had also asked to patent the actual mice, as well as all non-human mammals that have been modified to be vulnerable to cancer.

The patents have already been granted in all of these countries except Canada. But on
5 December, Canada's highest court denied Harvard's request to patent the mice. "A higher life form is not patentable because it is not a 'manufacture' or 'composition of matter," the court said in its ruling, which can't be appealed except through legislation.

The court did not rule out Harvard's claims on the process used to make the oncomouse, however, and the university says it will continue to pursue that claim.

The decision isn't seen as a huge setback for Harvard, because Canada is a relatively small part of the global market for the mouse. But it is expected to have major ramifications for life-sciences research and biotechnology in Canada. BIOTECanada, a biotechnology association, says the move will deter researchers from trying to develop research tools, such as transgenic animals, that could be useful for science and medicine.

But environmental groups welcomed the decision. "We think the court got it right," says Jo Dufay, campaign director for Greenpeace Canada. "These issues are so complex that they require full public debate, and that goes beyond a simple tinkering with the Canadian patent act." The court ruling is expected to lend impetus to a major overhaul of Canadian patent law, which parliament is expected to take on before the current government leaves office. 
astronomy and physics (see Nature 416, 775;2002).

The roadmap's top two recommendations for 'Einstein Great Observatories' were also ranked highly in the decadal review. These are the Laser Interferometer Space Antenna (LISA), a European-US space-based array designed to detect gravitational waves, and Constellation-X, a cluster of X-ray telescopes that would conduct high-resolution spectroscopy of energetic objects, including black holes.

The smaller, more focused Einstein Probes would be proposed and managed by scientists outside NASA, and would be launched every three to four years. The first three candidate missions cited in the roadmap are a Dark Energy Probe, an Inflation Probe to study the remnant radiation from the Big Bang, and a Black Hole Finder Probe.

Agency scientists and roadmap team members have already begun briefing Washington policy-makers on the plan, which would need funding beyond NASA's current \$3.6-billion annual budget for space science. All are well aware of the need to sell the new initiative. Public communication is "absolutely critical to winning support for Beyond Einstein", Kinney told the advisory meeting.

But space scientists are confident that the programme can deliver both cuttingedge science and ample public enthusiasm. "These are sexy topics," says Rocky Kolb, an astrophysicist at Fermilab in Batavia, Illinois, who chairs the SEU subcommittee.

\section{Paper trail reveals references go unread by citing authors}

Philip Ball

Many of the references cited in scientific papers have not been read by the authors citing them, according to an analysis of how errors in citations propagate through the literature.

It isn't easy to establish directly - and truthfully — whether citations have been read. So Mikhail Simkin and Vwani Roychowdhury, two electrical engineers at the University of California, Los Angeles, decided to estimate author diligence by checking how often errors in citation lists are passed on through other papers. They reckon that scientists who have looked up and read a paper they want to cite are unlikely to misprint it in their own articles. The pair concluded that four out of five authors had not done their homework (M. V. Simkin and V. P. Roychowdhury Preprint cond-mat/0212043 http://xxx.lanl.gov; 2002).

Simkin and Roychowdhury tracked all of the citations of a seminal 1973 paper on condensed-matter physics (J. M. Kosterlitz and D. J. Thouless J. Phys. C 6, 1181-1203; 1973). They looked at 4,300 citations of this paper and found that mis-citations of it were often identical to each other.

The probability of the same misprint occurring twice by chance is very small, so Simkin and Roychowdhury conclude that these errors are usually propagated by the reference being copied from someone else's citation list. The most common misprint appeared 78 times.

Based on the number of distinct misprints, the two researchers estimate that only 22-23\% of citations followed from a reading of the original paper. And they postulate that this is typical of the scientific literature as a whole.

Others agree that lazy citation is a real issue. Sidney Redner, a physicist at Boston University who has studied citation statistics, says that he thinks the latest estimate of original reading may be on the low side. "I might have guessed at about 50\%," he says, but he agrees with the qualitative conclusion that many citations are unread.

How much does it matter? The Office of Research Integrity (ORI) at the US health department has recently drawn criticism for probing into this kind of practice, which is considered by some to be a kind of low-key misconduct (see Nature 420, 253; 2002). If the problem is as widespread as the new study implies, it becomes harder to dismiss.

"I think it's very serious," says Kay Fields of the ORI, although she sees it as a matter of negligence rather than misconduct. Fields points out that failure to consult the original source can also propagate misconceptions about what the source's conclusions actually are. "It's poor research practice, for sure," she says.

\section{Radar array tests the atmosphere at pole position}

\section{David Cyranoski, Tokyo}

A three-metre radar antenna was among the equipment carried by Kaoru Sato as she set off for the South Pole late last month. Sato, who is based at the National Institute of Polar Research (NIPR) in Tokyo, will do a pilot assessment for a project that could make the Antarctic home to 1,000 such antennas set up in a circular array some $\mathbf{1 6 0}$ metres across.

The array would fill a major gap in our knowledge of the atmosphere, supporters of the project say. Already, radars around the world provide valuable data on phenomena such as atmospheric energy transfers. Researchers use the arrays to transmit radio waves. They then read their reflections to establish fluctuations in temperature, electron density and wind turbulence.

Such data offer information on how the different levels of Earth's atmosphere interact with each other and with the planet's climate - an important component in the study of global climate change and in understanding global weather circulation. But according to Masaki Ejiri, head of the NIPR project, global models badly need more information about such patterns in the little-studied region of the South Pole's atmosphere. "The unknowns far surpass those anywhere else," he says of the area.

A better understanding of energy transfer in the atmosphere will also help to answer several other questions, Ejiri says, including whether the current improvement in the

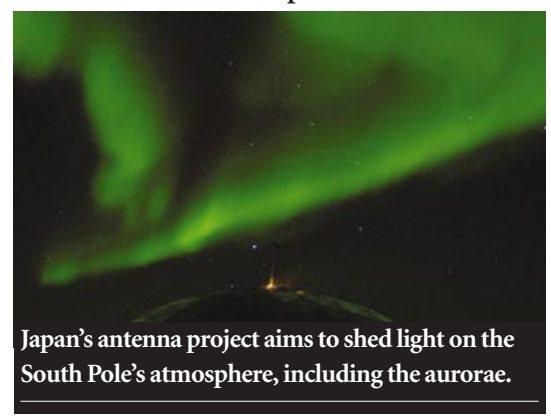

ozone layer's condition is likely to be shortlived, and how aurorae affect temperatures in the middle atmosphere.

But Sato's bold mission won't be easy to complete. On current estimates, the circular array would consume more power than Japan's entire South Pole research base, says Toru Sato, Kaoru's husband and an engineer at Kyoto University. Sato believes he can reduce this energy consumption by half.

Other technical problems remain to be solved. One is ensuring that the equipment can withstand the low temperatures in the region. Another is interference with the radar signal by other radio waves. Sato's work with the single antenna will seek to measure this extraneous 'noise' with a view to figuring out how best to neutralize it.

But the project's biggest long-term challenge is to win the tens of billions of yen it would cost to build. Ejiri says that he is "convinced that the project is valuable enough to overcome these difficulties". 